\section{Controle de formigas cortadeiras com Penicillium spp. proveniente de laranjas em decomposição: Aplicação em diferentes frequências avaliadas por agricultores}

\author{
Control of leaf-cutting ants with penicillium spp. from \\ decomposing oranges: application at different \\ frequencies evaluated by farmers
}

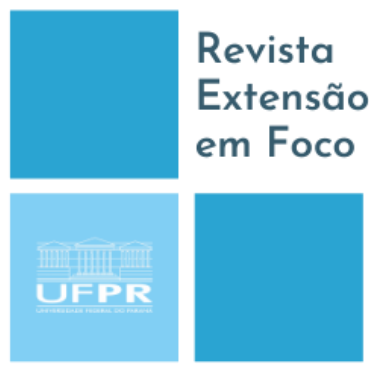

ISSN $2358-7180$

\author{
Alexandre De Donato ${ }^{1}$, Francis Alex Nunes ${ }^{2}$, Bianca Fraga Menezes ${ }^{3}$, André Barbosa Vargas ${ }^{4}$
}

RESUMO

As formigas cortadeiras são consideradas importantes pragas da agricultura brasileira, pois utilizam material vegetal como substrato para o cultivo do seu fungo simbionte. Dessa forma, fazem-se necessários métodos de controle para a contenção desses danos. O assentamento Roseli Nunes, situado em Brasil, Piraí, Rio de Janeiro, tem como um dos seus principais problemas fitossanitários o ataque por formigas cortadeiras dos gêneros Acromyrmex e Atta. Esta situação foi constatada a partir da atuação do Instituto Federal do Rio de Janeiro (IFRJ) campus Pinheiral, o qual vem auxiliando o assentamento por meio de um projeto que visa estabelecer um plano de desenvolvimento. O presente estudo consistiu em testar a eficiência de fungos do gênero Penicillium, no controle de formigas cortadeiras, obtidos de frutos em decomposição de variedades de laranjas. Foram realizadas diferentes frequências de aplicação e avaliadas empiricamente por 3 grupos de agricultores situados em diferentes áreas, isoladas a no mínimo $1 \mathrm{~km}$ uma da outra, dentro do assentamento. Os agricultores receberam capacitação para aplicar a calda fúngica e avaliar a sua eficiência, por meio da observação da atividade das formigas cortadeiras e dos danos causados às suas culturas por estas. Este método de controle biológico mostrou-se capaz de controlar a atividade de formigas cortadeiras quando aplicado mais de 20 vezes em um período de 50 dias. Contudo, a eficiência deste ainda não foi suficientemente estudada, e precisa de mais avaliações para ter a eficiência de sua utilização maximizada.

Palavras-chave: Penicillium. Controle biológico. Formigas cortadeiras. Extensão Rural.

\title{
ABSTRACT
}

\footnotetext{
${ }^{1}$ Mestre em Fitossanidade e Biotecnologia Aplicada. Instituto Federal de Educação, Ciência e Tecnologia do Rio de Janeiro (IFRJ), Pinheiral, RJ, Brasil. E-mail: alexandre.donato@ifrj.edu.br. Orcid: https://orcid.org/0000-0002-6514-2654.

${ }^{2}$ Mestre em Agricultura Orgânica. Secretaria Estadual de Agricultura, Pecuária, Pesca e Abastecimento do Estado do Rio de Janeiro (SEAPPA), Barra do Piraí, RJ, Brasil. E-mail: francisalex nunes@yahoo.com.br. Orcid: https://orcid.org/0000-0003-0671-3506.

${ }^{3}$ Doutora em Genética. Instituto Federal de Educação, Ciência e Tecnologia do Rio de Janeiro (IFRJ), Pinheiral, RJ, Brasil. E-mail: bmenezes@ biologia.ufrj.br Orcid: https://orcid.org/0000-0002-6544-9891

${ }^{4}$ Doutor em Ciências Ambientais e Florestais. Centro Universitário de Volta Redonda (UNIFOA), Volta Redonda, RJ, Brasil. E-mail: andrebvargas@yahoo.com.br. Orcid: https://orcid.org/0000-0002-8340-8217.
} 
Leaf-cutting ants are considered important pests in agriculture, as they use plant material as substrate for the cultivation of their symbiotic fungus. Thus, control methods are necessary to contain these damages. The Roseli Nunes settlement, located in Brazil, Piraí, Rio de Janeiro, has as one of its main phytosanitary problems the attack by leaf-cutting ants of the Acromyrmex and Atta genera. This situation was verified through the work of the Federal Institute of Rio de Janeiro (IFRJ), which has been helping the settlement through a project that aims to establish a development plan. The present study consisted of testing the efficiency of fungi of the genus Penicillium obtained from decomposing orange fruits with different application frequencies, in the control of leaf-cutting ants, which was evaluated empirically by 3 groups of farmers located in different areas, isolated at least $1 \mathrm{~km}$ from each other, within the settlement. The farmers received training to apply fungal syrup and evaluate its efficiency, by observing the activity of leaf-cutting ants and the damage caused to their crops by them. In the application of fungal syrup and evaluation of the activity of leaf-cutting ants, after its application, through observations of their activity and evaluation of the damages caused to their cultures. This method of biological control was able to control the activity of leaf-cutting ants when applied more than 20 times in a period of 50 days. However, its efficiency has not yet been sufficiently studied, and it needs more evaluations to maximize its efficiency.

Keywords: Penicillium. Biological Control. Leaf-cuting ants. Rural Extension.

\section{INTRODUÇÃO}

As formigas cortadeiras são insetos polífagos que se encontram na Ordem Hymenoptera, família Formicidae, subfamília Myrmicinae, tribo Attini. Seu hábito social é fortemente estabelecido, organizando-se com divisão de atividades bem definidas por meio de castas. Ao longo de sua evolução, desenvolveram o hábito de cultivar um fungo simbionte do qual se alimentam, sendo este da espécie Leocoagaricus gongylophorus, família Agaricales e ordem Basidiomycota. As principais espécies que vem se destacando por atacarem plantas em áreas agrícolas, florestais e urbanas são: Atta laevigata, Atta sexdens rubropilosa, Acromyrmex disciger, Acromyrmex niger e Acromyrmex crassipinus. Os danos causados têm maior destaque nas fases de pré-corte (áreas de reforma ou condução da floresta) e imediatamente após o plantio ou no início da condução de brotação (GIESEL et al., 2020).

O manejo de formigas invasoras engloba diversos métodos de controle que tentam reduzir a velocidade de propagação e mitigar seus impactos para o homem e o meio ambiente. O método natural mais sustentável consiste no controle biológico clássico, onde inimigos naturais são introduzidos e se estabelecem no agroecossistema para reduzir a população da espécie alvo. Formigas cortadeiras podem ser controladas utilizando métodos químicos, biológicos, mecânicos e culturais. $\mathrm{O}$ emprego mecânico manual praticamente não é utilizado em função de só ser aplicável a pequenas áreas e a ninhos com menos de quatro meses (SILVA et al., 2020). A atividade herbívora deste grupo de formigas pode representar e acarretar problemas econômicos em cultivos comerciais como na cafeicultura (DA SILVA ARAÚJO et al., 2015) e em florestas comerciais de Eucalyptus spp. e Pinus spp. (SANTOS, 2017). 
As formigas produzem substâncias antibióticas que visam à proteção do fungo simbionte, efetuando inclusive uma inspeção do material vegetal trazido previamente à incorporação deste ao ninho, de modo a evitar sua contaminação. Outro hábito das colônias de formigas cortadeiras que desfavorece o controle biológico, especialmente a utilização de fungos entomopatogênicos, é a execução de procedimentos de higiene por parte das operárias da colônia, no qual o material danoso, após o reconhecimento pelas formigas, é removido do ninho (formigas doentes, mortas, materiais vegetais contaminados, etc). (BUENO, 2020).

As formigas cortadeiras são insetos praga polífagos, isto é, conseguem se alimentar de diversos gêneros de plantas (CHAMY, 2017). As que constroem os maiores ninhos são às pertencentes aos gêneros Atta (Saúvas) e Acromyrmex (Quenquéns), as quais podem possuir milhões de operárias em seus ninhos e o seu prejuízo é estimado em bilhões de dólares em todo o planeta. Dentre as diversas famílias de plantas atacadas, podemos citar como exemplos as famílias Aizoaceae, Marattiaceae, Amaranthaceae, Metastomateceae, Bignonaceae, Moraceae, Brassiceae, Passifloraceae, Caryophyllaceae, Rosaceae, Cucurbitaceae, Rutaceae, Euphorbiaceae, Salicaceae, Fabaceae e Solanaceae, (GIESTAS et al., 2020; SANTOS, 2017).

O controle mais difundido para combater formigas cortadeiras consiste na utilização de iscas granuladas, que possuem o formicida sulfluramida como ingrediente ativo mais comum, onde a sua ação lenta e necessidade de baixas concentrações faz com que este produto seja considerado menos nocivo à saúde humana e meio ambiente. Este método de controle se sobrepôs ao uso de termonebulizador ou polvilhadeira, devido ao seu menor risco de contaminação ambiental e do operador, além de ter um modo de aplicação mais prático (DE BRITTO, 2016). A termonebulização é a produção de uma fumaça tóxica aplicada diretamente nos olheiros por meio da queima de querosene ou óleo diesel, a qual libera também o formicida. Olheiros são cavidades no solo que servem para entrada e/ou saída de formigas, seus alimentos, dejetos, ou mesmo a entrada de ar no formigueiro. Contudo, no caso específico da espécie de formiga cortadeira denominada Acromyrmex rugosus, o controle com iscas granuladas é pouco eficaz, visto que estas formigas têm dificuldade de transportar as iscas até o interior de seus ninhos, uma vez que estas possuem uma estrutura corpórea bastante reduzida (SANTOS, 2017; CHAMY, 2017).

Dentre as formas de controle químico, os à base de sulfluramida são os mais utilizados para o controle populacional deste inseto, devido ao baixo custo e rápida resposta. Porém, insumos e pesticidas podem intoxicar seres humanos e animais e/ou atingir grupos de insetos 
predadores naturais, originando danos ambientais, além de influenciar na dinâmica da flora e fauna local (BURATTO et al., 2017; GIESTAS et al., 2020). Todavia, apesar desta caracterização de "praga", as formigas cortadeiras não trazem apenas prejuízos, mas também benefícios com atuações em importantes processos ecológicos, como a ciclagem de nutrientes, nos mais diversificados ecossistemas em que se encontram. Neste sentido, a dispersão de controle biológico tem se destacado como um método de controle que visa manter, de modo duradouro e estável, abaixo do nível de dano econômico, a espécie em questão, por meio da utilização de inimigos naturais, os quais podem ser predadores, parasitas, ou patógenos (DA SILVA ARAÚJO et al., 2015; FONSECA, 2019). Em tese, os inimigos naturais seriam capazes de se espalhar naturalmente pela área, alcançando inclusive áreas inacessíveis a humanos e reduzindo as taxas de crescimento e disseminação do inseto alvo, predando-o (OI et al., 2015).

Oi e colaboradores (2015) avaliaram diversos inimigos naturais, entre vírus (SINV 1, 2 e 3) bactérias e fungos entomopatogênicos, vespas parasitas e vespas que decapitam formigas operárias. A maioria demonstrou um bom resultado em condições de laboratório; contudo, em condições de campo, o controle efetuado sobre formigas cortadeiras foi pouco significativo em todos eles, ou viável apenas para áreas muito pequenas, como no caso de alguns vírus. Divergências como esta (entre resultados "de laboratório" e os resultados de campo, também denominados pelos agricultores como resultados "de verdade"), tem sido uma das importantes razões para a menor capilaridade de métodos científicos criados sem a participação dos agricultores.

A Extensão, como ferramenta institucional, precisa trabalhar com os grupos-alvos (principalmente os caracterizados como vulneráveis ou com qualquer outra necessidade) por meio de articulação e mobilização em prol da melhoria nas condições sociais, sendo auxiliada pelos agentes que trabalharão com a atividade extensionista. Durante algumas décadas, dentro do processo histórico de complexificação e cientificação dos saberes, os técnicos de campo ligados à agricultura foram vistos como meros difusores de tecnologia, limitados em suas capacidades de criação e de conhecimento. Contudo, no atual momento pós-modernizado, são exigidas novas competências ligadas a conteúdo, valores e forma de conhecimento, além do questionamento do conteúdo social, da técnica e do autoritarismo dos métodos de persuasão, em virtude de novos desafios impostos a estes profissionais por meio de conflitos na área rural e limites ambientais (SERRÃO, 2020; LIMA, 2018). 
Uma sistematização partilhada de saberes ou de técnicas seria uma alternativa que permite superar o tecnicismo além de possibilitar ao profissional a contribuição por meio de técnicas e procedimentos alternativos para auxiliar na produção de alimentos. E por uma questão ética, de validação e apuração da percepção por parte dos técnicos, a devolução dos dados à comunidade se faz necessário de forma rápida, podendo ser também criativa para favorecer o debate. Isso permite que ambos os lados (técnicos e comunidade) possam notar de forma explícita os equívocos oriundos das distintas percepções (COELHO, 2005).

Desde seu surgimento, a extensão rural representou muito mais que um mero conjunto de ideias veiculadas, demonstrando uma grande preocupação em saber como o processo de implementação dessas ideias se daria. Isso justifica sua presença histórica em debates pedagógicos, mesmo se tratando de uma instituição efetivada majoritariamente pela educação não formal. Mas nota-se a importância, enquanto instituição pedagógica, da retomada dos debates teórico-metodológicos que possam subsidiar seus processos de intervenção (JUNIOR, 2020).

O presente estudo foi realizado no assentamento Roseli Nunes, que tem como um dos seus principais problemas fitossanitários o ataque por formigas cortadeiras dos gêneros Acromyrmex e Atta. A maioria dos produtores dispõe de pouco capital, o que por si só já dificulta a utilização de iscas granuladas. Vale destacar que muitos dos produtores são adeptos da filosofia de produção agroecológica, o que também os têm levado a utilizar métodos de controle mecânicos, como barreiras para proteger a copa das árvores, escavação do formigueiro, dentre outros. Esta situação foi constatada a partir da atuação do Núcleo de Estudos e Atuação em Agroecologia (NEAA) do Instituto Federal de Educação, Ciência e Tecnologia (IFRJ) do campus Pinheiral, que vem auxiliando o assentamento a partir de um plano de desenvolvimento desenvolvido no projeto do IFRJ (NUNES et al., 2017).

Sabe-se que fungos do gênero Penicillium são comuns no solo, não tendo importância como agente etiológico vegetal, pois se desenvolvem em plantas e frutos em estágio de decomposição, podendo produzir milhões de esporos em alguns dias de desenvolvimento (BALLESTER et al., 2017; SIQUEIRA; SALOMÃO, 2016). O controle de formigas cortadeiras utilizando Penicillium spp. oriundos de cascas de laranjas em estágio avançado de decomposição, surgiu empiricamente, a partir da atuação de produtores agroecológicos na busca por alternativas de controle e manejo de formigas cortadeiras, e seus resultados mostraram uma redução na atividade de forrageamento em ninhos de Acromyrmex spp 
(MOREIRA \& TALACZ, 2013). Em razão dos fatos expostos, o objetivo do presente estudo consistiu na avaliação da eficiência de Penicillium spp. obtidos de frutos de laranja em decomposição, em diferentes frequências de aplicação, no combate a formigas cortadeiras.

\section{MÉTODOS}

O estudo foi realizado no assentamento Roseli Nunes, situado no município de Piraí, localizado ao sul do Estado do Rio de Janeiro entre as coordenadas geográficas $22^{\circ} 35^{\circ}$ e $22^{\circ}$ $35^{\circ} \mathrm{S}$ e $43^{\circ} 55^{\circ}$ e $43^{\circ} 56^{\circ} \mathrm{W}$. Os terrenos do assentamento variam de planos a ondulados, abrangendo áreas com declividade variando de $3^{\circ}$ a $25^{\circ}$. As formações vegetais dominantes na região são áreas abertas de pastagens, florestais, frutícolas, hortícolas e diversos fragmentos florestais remanescentes da Mata Atlântica. O clima é Ws, de acordo com a classificação de Köppen, com estação chuvosa no verão e seca no inverno, com temperatura média máxima de $29,1^{\circ} \mathrm{C}$ em fevereiro, e temperatura média mínima de $20,1^{\circ} \mathrm{C}$ em julho. A pluviosidade média anual é de $1.238,5 \mathrm{~mm}$, ocorrendo abundantes chuvas em fevereiro e escassas em julho (BAYLÃO JUNIOR et al., 2008).

\section{Delineamento das atividades do experimento}

Os extensionistas reuniram-se com a maior parte das 23 famílias assentadas que participaram das primeiras reuniões do ano de 2018. Nesta reunião, explicou-se que um novo método experimental poderia controlar as formigas cortadeiras da região, de eficiência ainda não consolidada do ponto de vista científico, porém com baixíssimo custo (ou custo zero se não considerarmos o tempo de trabalho das famílias como custo) e sem nenhum impacto ambiental. Um total de oito famílias aceitou fazer parte da experiência científica empírica, enquanto os demais ou não apresentavam problemas com formigas cortadeiras ou tinham começado tratamentos químicos recentemente. Após uma longa discussão, os agricultores decidiram em quais famílias/propriedades cada tratamento seria aplicado.

Foram estipuladas três áreas de tratamentos para a coleta de dados e amostragem dos ninhos, abrangendo a área dos formigueiros em metro quadrado $\left(\mathrm{m}^{2}\right)$, quantidade de olheiros, declividade, área do tratamento e espécie predominante. No tratamento 0 , a calda fúngica não foi aplicada, e o tratamento ocorreu nas propriedades vizinhas àquelas onde os tratamentos com a calda fúngica foi utilizada, tendo sido feita a avaliação por cinco famílias, totalmente distintas das famílias dos outros tratamentos. No tratamento 1 , foi aplicada a calda fúngica com uma 
frequência de 10 ou 13 dias entre cada aplicação, tendo sido feitas quatro aplicações ao longo dos 50 dias de tratamento. Foi estimado um número de olheiros situado entre 150 e 200, os quais totalizavam uma área aparente estimada entre 200 e $300 \mathrm{~m}^{2}$. A espécie de formiga cortadeira predominante na área foi Atta laevigata, popularmente conhecida como saúva cabeça-de-vidro. A área total deste tratamento foi estimada em 1,14 ha. Esta área tratada possuí terreno predominantemente plano, com declividade máxima estimada em cerca de $5 \%$.

No tratamento 2, foi aplicada a calda fúngica com uma frequência de 2 ou 3 dias entre cada aplicação, tendo sido feitas 20 aplicações ao longo dos 50 dias de tratamento. Foi estimado um número de olheiros situado entre 100 e 150, os quais totalizavam uma área aparente estimada entre 100 e $150 \mathrm{~m}^{2}$. A espécie de formiga cortadeira predominante na área foi Atta sexdens, popularmente conhecida como saúva limão. A área total do experimento foi estimada em 1,45 ha. Esta área tratada se divide em duas glebas, uma com terreno predominante plano, com declividade estimada inferior a 5\%, e outra com área ondulada e declividade situada entre 10 e $20 \%$.

No tratamento 3, foi aplicada calda fúngica com uma frequência de 1 dia entre cada aplicação, tendo sido feitas 50 aplicações ao longo dos 50 dias de tratamento. Foi estimado um número de olheiros situado entre 50 e 100, os quais totalizavam uma área aparente estimada entre 50 e $80 \mathrm{~m}^{2}$. A espécie de formiga cortadeira predominante na área foi Atta laevigata. A área total do experimento foi estimada em 0,65 ha. Esta área tratada possuí terreno predominantemente plano, com declividade máxima estimada em cerca de $5 \%$.

A área do tratamento 1 se situa a dois $\mathrm{km}$ da área onde foi efetuado o tratamento 2 , e a três $\mathrm{km}$ da área onde foi efetuado o tratamento 3. Enquanto a área em que foi efetuado o tratamento 2 se situa a quatro $\mathrm{km}$ da área onde foi efetuado o tratamento 3 . Na área do tratamento 1, a área tratada foi inferior a $10 \%$ da área total do lote cultivado, enquanto que no tratamento 2 situou-se entre 20 e $30 \%$ e, no tratamento 3, entre 40 e $50 \%$. Em todas as áreas, havia um percentual expressivo de mata nativa circundante ao longo dos lotes, o que favorece o isolamento entre as regiões de tratamento e impossibilita a interferência de um tratamento sobre o outro.

Também foi incluído um tratamento 0 , denominado de testemunha, que serviu de controle do experimento. Em todos os tratamentos, a avaliação da eficácia foi efetuada por diferentes agricultores, sendo feita pela respectiva família que cultivava a propriedade onde o tratamento foi aplicado. Em nenhum caso uma mesma família avaliou diferentes tratamentos, 
assim como não houve uma mesma propriedade recebendo mais de um tratamento. Contudo, a metodologia de avaliação ensinada aos agricultores foi a mesma para todas as famílias nas diferentes áreas tratadas, aumentando a homogeneidade entre as áreas e tratamentos.

Nestes tratamentos, as formigas cortadeiras foram capturadas por amostragem em diferentes dias, horários e parcelas das áreas do experimento, sendo posteriormente montadas em via seca e identificadas ao nível de gênero de acordo com as chaves dicotômicas propostas por Baccaro e colaboradores (2015). A identificação ao nível de espécie, quando possível, foi realizada pela utilização de chaves específicas para cada gênero.

\section{Preparo da calda e avaliação da percepção de controle pelos agricultores}

As laranjas em estado de decomposição foram maceradas e colocadas em baldes de 10 litros com água em uma proporção de 3 partes de água para 1 parte de macerado em volume $(25 \% \mathrm{em} \mathrm{v} / \mathrm{v})$ e deixadas para fermentar durante 7 dias. Essa calda fermentada na concentração de $25 \%$ foi despejada diretamente em cada olheiro dos formigueiros demarcados, adaptado de Moreira e Talacz (2013). Em média, foram utilizados $200 \mathrm{~mL}$ da calda por olheiro por aplicação, podendo chegar a $500 \mathrm{ml}$ para olheiros com mais de $4 \mathrm{~m}^{2}$ de área aparente (área de terra solta típica dos formigueiros calculada pela multiplicação das duas maiores extensões medida em ambas as dimensões), conforme avaliação do agricultor.

Notas de 0 a 10 foram obtidas a partir da percepção empírica dos agricultores em relação ao forrageamento e atividade das formigas cortadeiras antes e após 7, 21 e 35 dias de tratamento. Os agricultores foram capacitados para realizarem as observações e anotações dos níveis de injúria das plantas, a partir de seu nível de desfolha, de acordo com a metodologia de Casa e colaboradores (2007). Foi realizado o teste de Friedman, uma análise não-paramétrica utilizada para comparar dados amostrais vinculados, ou seja, quando o mesmo indivíduo é avaliado mais de uma vez. O teste de Friedman não utiliza os dados numéricos diretamente, mas sim os postos ocupados por eles após a ordenação feita para cada grupo separadamente. Após a ordenação, é testada a hipótese de igualdade da soma dos postos de cada grupo. As análises estatísticas foram realizadas no programa Systat 13 (Systat Software, Chicago, IL, U.S.A.).

\section{RESULTADOS E DISCUSSÃO}


Os serviços de pesquisa e extensão rural são fundamentais para disseminar tecnologias e aumentar quantitativamente e qualitativamente a produção agropecuária, contudo, a falta de envolvimento dos profissionais acadêmicos com agricultores (o que têm contribuído para acusações de "resultados artificiais de pesquisa", que quando aplicados na "agricultura de verdade" podem não funcionar) têm muitas vezes gerado tecnologias inadequadas à realidade de pequenos produtores de alimentos, especialmente nos países em desenvolvimento. O modo como os agricultores percebem as tecnologias influencia sua decisão de adotá-las ou não. A participação dos agricultores na geração e disseminação de tecnologias agrárias é fundamental para que estas tecnologias se desenvolvam de modo compatível à realidade e necessidade do agricultor, aumentando significativamente as chances da atividade de pesquisa e extensão ser bem-sucedida, isto é, se tornar parte da rotina produtiva de uma proporção considerável dos produtores de alimentos (COME, 2019).

A Extensão Rural Agroecológica caracteriza-se como um esforço para a realização de uma intervenção participativa planejada que possibilite o desenvolvimento rural sustentável, considerando o saber local junto ao científico como base para implementação de agriculturas alternativas biodiversas e amparadas por questões socioculturais. É importante que o Extensionista possa reconhecer o conhecimento popular oriundo das experiências, impulsionadas pelas necessidades históricas e estilos de vida específicos, reconstruindo o Sistema Agrícola Tradicional com uso de conhecimentos tradicionais acumulados, sem com isso negar a utilidade da ciência convencional, demonstrando a importância da integração entre agentes externos e os agricultores, pautado na racionalidade e na lógica própria de cada um dos estilos de agricultura (CAPORAL, 2009).

Espera-se que a participação dos pequenos agricultores no experimento realizado pelo presente trabalho produza um maior impacto na credibilidade e difusão da técnica agroecológica, pois de acordo com a avaliação de Schneider (2020), Da Silva e colaboradores (2020), Oliveira (2020) e Lins e Pires (2018), é importante valorizar os saberes populares e permitir a participação ativa de agricultores e produtores rurais na construção do saber. A figura 1 retrata o momento em que uma das agricultoras está recebendo o treinamento:

Figura 1 - Agricultora do tratamento 3 recebendo instruções sobre como preparar a calda e avaliar os resultados no Assentamento Roseli Nunes, Piraí, Rio de Janeiro. 


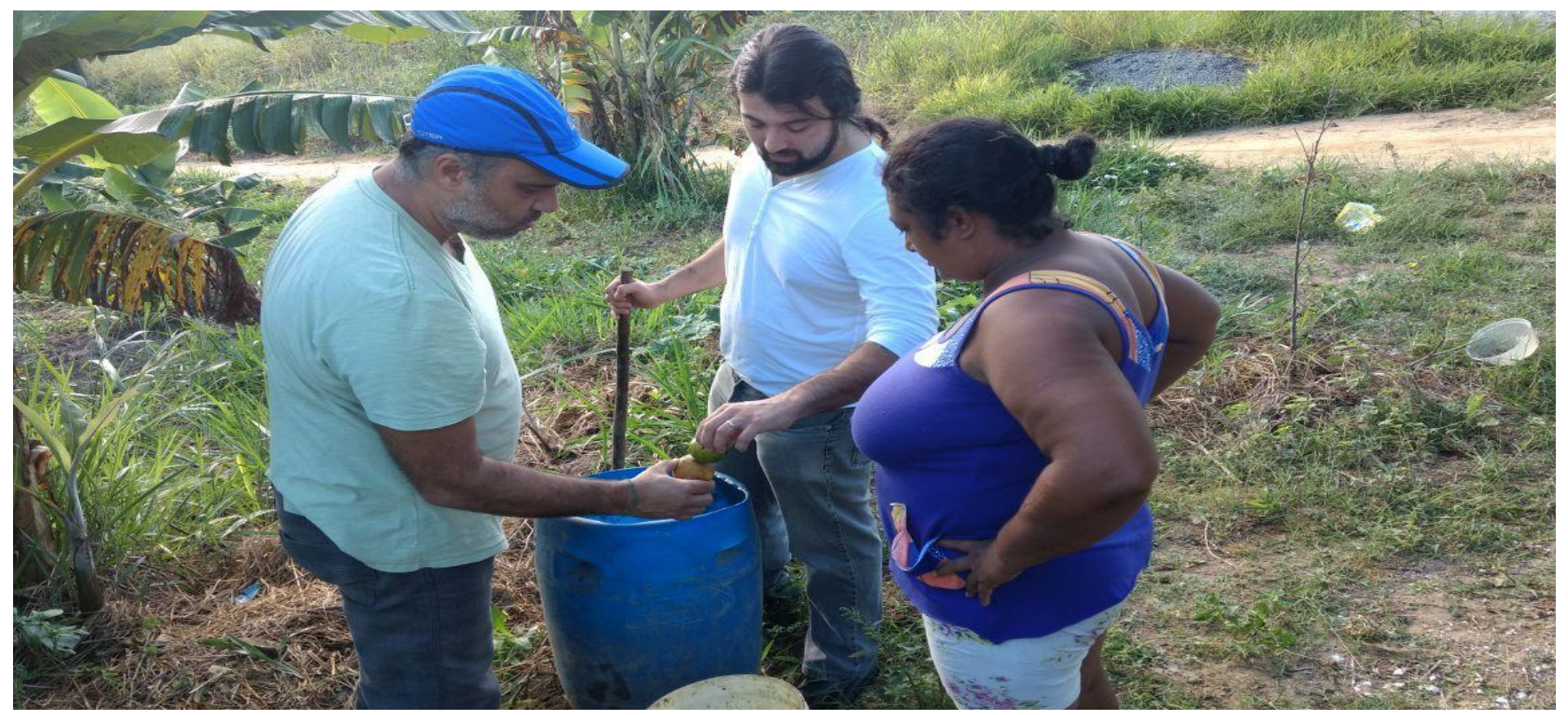

Fonte: Autoria própria.

Figura 2 - Área de trabalho do tratamento 3 no Assentamento Roseli Nunes, Piraí, Rio de Janeiro.

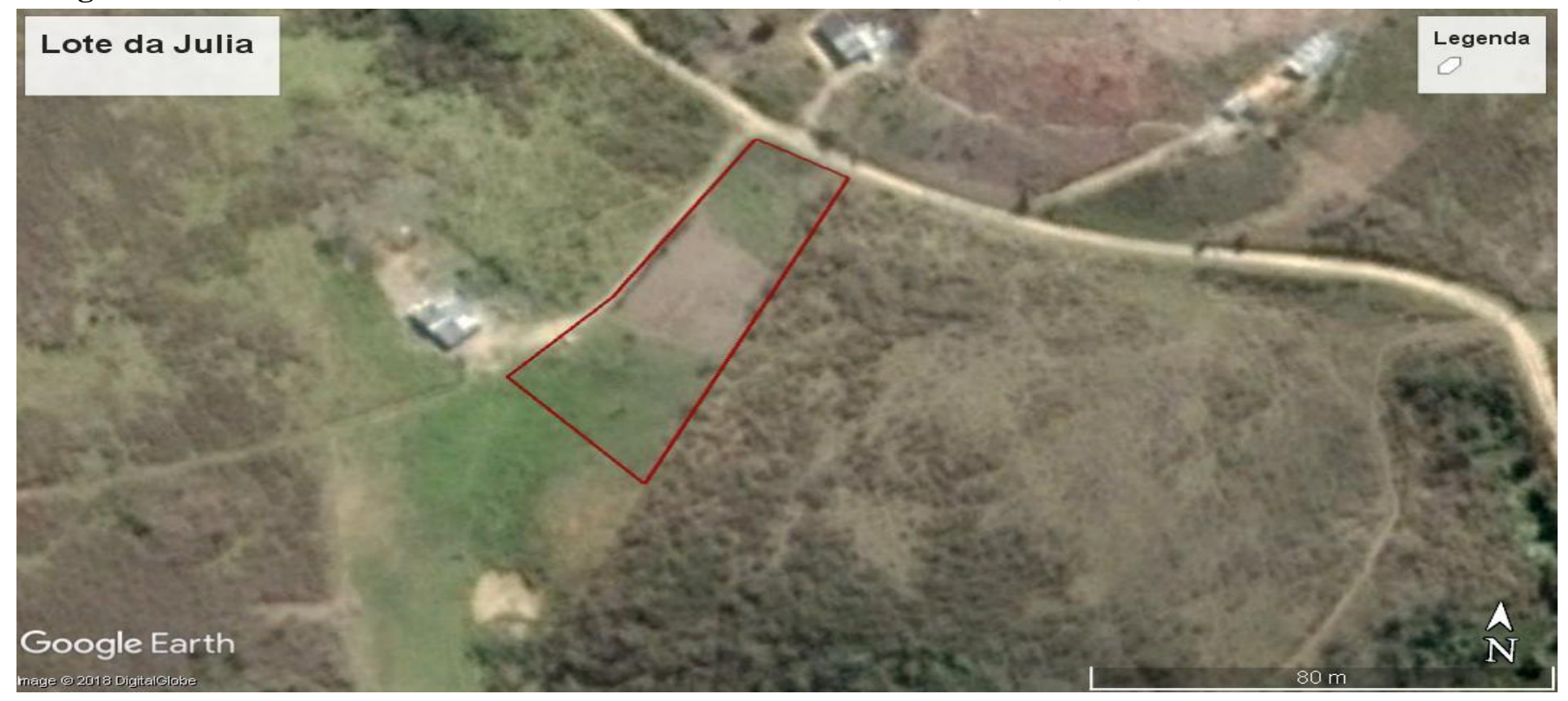

Fonte: Autoria própria.

A tabela 1 apresenta as notas (0-10) dos agricultores, bem como a área do terreno, declividade, altitude e espécie predominante por tipo de tratamento realizado. Foi detectada uma diferença significativa na diferença entre os tipos de tratamento $\left(X^{2}=7,9 ; P=0,02\right)$, levando em consideração as três avaliações (explicitadas nos materiais e métodos). Este resultado demonstra que a frequência de dias entre cada aplicação exerce um efeito nos resultados observados pelos agricultores. 
Tabela 1 - notas dos agricultores, área aparente dos formigueiros no terreno $\left(\mathrm{m}^{2}\right)$, declividade, altitude, NO (número de olheiros), A.trat (ha) (área tratada e, hectares) e espécie (espécie predominante por tratamento realizado) por tipo de tratamento (Trat 1, 2 e 3 ).

\begin{tabular}{|c|c|c|c|c|c|c|c|c|}
\hline & Nota 1 & Nota 2 & Nota 3 & Área (m²) & Declividade & NO & A.trat(ha) & Espécie \\
\hline $\begin{array}{l}\text { Trat } \\
\text { o }\end{array}$ & 0 & 0 & 0 & 1000 a 1200 & Plano/Ondulado & $\begin{array}{c}400 \mathrm{a} \\
500\end{array}$ & 4,8 & $\begin{array}{l}\text { Atta sexdens } \\
\text { e Atta } \\
\text { laevigatta }\end{array}$ \\
\hline $\begin{array}{l}\text { Trat } \\
1\end{array}$ & 0 & 2 & 4 & 200 a 300 & Plano & $\begin{array}{c}150 \mathrm{a} \\
200\end{array}$ & 1,14 & $\begin{array}{l}\text { Atta } \\
\text { laevigatta }\end{array}$ \\
\hline $\begin{array}{l}\text { Trat } \\
2\end{array}$ & 0 & 6 & 8 & 100 a 150 & Ondulado & $\begin{array}{c}100 \mathrm{a} \\
150\end{array}$ & 1,45 & Atta sexdens \\
\hline $\begin{array}{l}\text { Trat } \\
3\end{array}$ & 2 & 7 & 9,5 & 50 a 80 & Plano & 50 a 100 & 0,65 & $\begin{array}{l}\text { Atta } \\
\text { laevigatta }\end{array}$ \\
\hline
\end{tabular}

Nos primeiros 3 dias após a primeira aplicação, em todos os tratamentos, foi observado o seguinte comportamento: As formigas removeram uma quantidade expressiva de terra do interior do formigueiro; provavelmente na tentativa de descontaminar o ninho, o que corrobora com Nagamoto e colaboradores (2011). Outro comportamento igualmente observado em todas as áreas, foi o encerramento total das atividades em alguns olheiros, redução da atividade em outros e o surgimento de outros olheiros em pontos situados a 100 metros ou mais dos olheiros extintos. De acordo com Dornelas e colaboradores (2017) e Polezel (2017), este comportamento era esperado, pois há uma série de outros fungos também considerados antagonistas do fungo simbionte de formigas cortadeiras, assim como diversos outros organismos. Consequentemente, as formigas cortadeiras, ao longo de seus milhões de anos de coevolução com o fungo simbionte, desenvolveram hábitos profiláticos para evitar a entrada de potenciais patógenos aos seus fungos simbiontes; tais quais limpar o material vegetal coletado, reinocular o micélio do fungo mutualista no substrato vegetal constantemente coletado, e remover fragmentos dos jardins de fungos com a presença de esporos germinados de outros fungos, fisicamente, ou quimicamente com suas glândulas antimicrobianas.

No tratamento 1, foram efetuadas quatro aplicações ao longo dos 50 dias de tratamento, onde a maior nota atribuída foi quatro, em uma escala de 0 a 10, sendo 0 nenhum efeito e 10 
um efeito excelente. $\mathrm{O}$ agricultor, de acordo com a capacitação recebida, realizou essa atribuição em função do quanto este observou redução na atividade de forrageamento das formigas, sua abundância e nos danos provocados a sua lavoura.

No tratamento 2, onde foram feitas 20 aplicações ao longo dos 50 dias de tratamento, a maior nota atribuída foi 8. O agricultor, para avaliar a eficiência do tratamento, recebeu as mesmas instruções dos tratamentos 1 e 3 . No tratamento 3, onde foram feitas 50 aplicações ao longo dos 50 dias de tratamento, a maior nota atribuída foi 9,5.

Durante a reunião de planejamento onde foram selecionados os agricultores que participariam do experimento, as cinco famílias que aceitaram dispor suas propriedades ao tratamento zero (testemunha) alegaram que não pretendiam, no curto prazo, efetuar algum cultivo com fins comerciais.

As demais famílias que recusaram o convite para participar do experimento, ou não tinham problemas com formigas cortadeiras, ou utilizaram tratamento químico recentemente ou se recusaram em virtude do tratamento "demorar muitos dias a funcionar", o que as fez preferirem utilizar métodos químicos de controle, ainda que com custo mais elevado.

Todos as famílias que aceitaram aplicar a calda fúngica e realizar as avaliações de eficácia do método de controle demonstraram um certo receio em "errar na nota de avaliação" ou "ver se o remédio funcionou". Foi necessário explicar que o método de avaliação da metodologia a ser aplicada é de fato empírico, e que variações são intrínsecas aos tratamentos e devem ser minimizados de acordo com a metodologia.

Cerca de um ano após a conclusão do experimento, dentro do IFRJ campus Pinheiral, foi realizado um seminário cujo título era "Seminário Agroecológico sobre a saúde das plantas: formigas cortadeiras" onde esta técnica foi relatada para mais de 80 agricultores que participaram presencialmente do evento, além de outros participantes, como alunos, professores e extensionistas. Considerando que Pinheiral-RJ e suas cidades vizinhas têm menos de 300 mil habitantes, dos quais a imensa maioria não é agricultora, e considerando também que quase 200 pessoas se inscreveram no evento, pode-se considerar que o interesse dos agricultores da região no controle de formigas cortadeiras é muito grande.

Os resultados de outros experimentos que utilizaram fungos ou outros organismos no controle de formigas cortadeiras são diversos. Palma (2016) avaliou a patogenicidade de 4 espécies de fungos sobre formigas cortadeiras e o grau de atratividade de iscas confeccionadas 
com diferentes substratos. As quatro espécies avaliadas foram: Aspergillus flavus (Trichocomaceae), Paecilomyces lilacinus (Trichocomaceae), Trichoderma harzianum (Hypocreaceae) e Cladosporium sp. (Davidiellaceae), e os substratos avaliados foram extratos em pó obtidos a partir de folhas e/ou frutos das seguintes espécies vegetais: Acalypha wilkesiana (Euphorbiaceae), Anadenanthera macrocarpa (Fabaceae), Rosa chinensis var. mínima, Citrus sinensis (Rutaceae), Triticum aestivum (Poaceae), cujos nomes populares são, respectivamente, Acalifa, Angico-vermelho, Mini rosa, laranjeira (neste caso o bagaço do fruto também foi utilizado) e Trigo. Após esta avaliação, conclui-se que apenas os isolados de

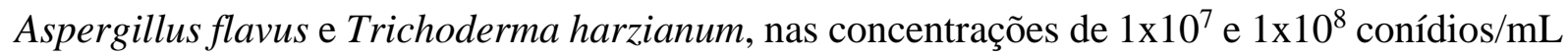
foram patogênicos (em condições experimentais de laboratório) e todas as iscas formicidas testadas apresentaram igual atratividade, contudo o tempo para contato e início de carregamento das iscas do angico-vermelho foi menor que os demais.

Canali (2017) em um estudo cujo objetivo foi estudar o potencial de fungos entomopatogênicos no controle biológico de formigas cortadeiras, concluiu que algumas linhagens de fungos (Beauveria bassiana, Isaria farinosa, Aspergillus nomius e Purpureocillium lilacinum) são particularmente promissoras para o desenvolvimento de bioinseticidas, e que a associação entre B. bassiana e A. nomius é especialmente patogênica, uma vez que atua contra formigas e também contra o fungo mutualista. Já a associação entre $B$. bassiana e I. farinosa, apesar de não ter apresentado relação antagonista frente ao mutualista, causa acentuada mortalidade entre as formigas.

$\mathrm{O}$ antagonismo de isolados fúngicos pertencentes ao gênero Trichoderma sobre o crescimento do fungo que alimenta as formigas cortadeiras (Leucoagaricus gongylophorus) foi testado por Nascimento e colaboradores (2017). Todos os sete isolados testados inibiram o crescimento do fungo simbionte. Contudo, esta eficiência ocorreu apenas em laboratório, não tendo sido feitos testes em condições de campo.

Freitas e colaboradores (2016) verificaram a atividade antagonista de 160 bactérias endofíticas de plantas da Amazônia contra microrganismos presente no ninho de formigas cortadeiras (Atta sexdens), concluindo, entre outras coisas, que em condições de laboratório, 38 bactérias demonstraram ter atividade antagônica contra L. gongylophorus, o que sinaliza uma possibilidade de avanço no sentido da obtenção de uma bactéria capaz de controlar formigas cortadeiras por meio da redução do fungo que serve como alimento a estas. 
Dornelas e colaboradores (2016) isolaram e identificaram fungos filamentosos associados às operárias de Atta sexdens e Atta laevigata, testando posteriormente a sua patogenicidade contra operárias da formiga A. sexdens. Foi possível identificar os fungos Metarhizium anisopliae, Aspergillus flavus, Acremonium sp., Colletotrichum sp., Fusarium solani, e A. niger. Ao final do estudo, concluíram que as espécies A. flavus, A. niger e $M$. anisopliae tem potencial de exploração para uso no controle biológico contra formigas cortadeiras, por apresentarem entomopatogenicidade.

Outro estudo, como o de Dornelas e colaboradores (2017) testaram a atividade antagonística, em relação ao fungo simbionte cultivado pelas formigas cortadeiras, de isolados do fungo Trichoderma spp, tendo avaliado também a ação do sulfato de zinco (ZnSO4), em função de sua possível ação imunossupressora sobre as formigas. Este estudo concluiu que o fungo Trichoderma spp, associado ao $\mathrm{ZnSO}$, foi capaz de reduzir a população do fungo simbionte, mas não houve mortalidade de formigas, apesar de a substância química ter provocado alterações no sistema autoimune das formigas cortadeiras. A viabilidade econômica e técnica da utilização de Trichoderma spp, em nível de campo no controle biológico de formigas cortadeiras, não foi avaliada.

Os Núcleos de Estudos em Agroecologia proporcionaram um aumento significativo de tecnologias e conhecimentos científicos aplicados a cultivos agroecológicos, visto que estes vêm se tornando pontos aglutinadores de pesquisas, formação e ações em agroecologia. Ao possibilitar a aproximação de agentes acadêmicos/técnicos com saberes populares (agricultores) e tradicionais, os conhecimentos científicos são articulados com o saber popular, o que aumenta a adequação do conhecimento/tecnologia à realidade dos agricultores, aumentando por conseguinte a sua aplicação (BERALDO et al., 2018).

Portanto, pode-se constatar que a metodologia de controle do presente trabalho pode ser promissora no controle de ninhos não somente de espécies do gênero Acromyrmex, conforme constatado por Moreira e Talacz (2013), mas também para espécies do gênero Atta. Outro fator que favorece o método de controle avaliado pelo presente trabalho é o fato de serem raros os relatos de reprodução sexuada deste fungo simbionte que alimenta as formigas cortadeiras, fator que propicia a sua baixa variabilidade genética, tornando-o mais vulnerável a infecções por outros competidores, como é o caso dos fungos do gênero Penicillium spp (MEIRELLES et al., 2015). Especificidades para cada espécie devem ser avaliadas, já que as espécies conhecidas como formigas cortadeiras (tribo: Attini), principalmente as espécies dos gêneros Acromyrmex 
e Atta, apresentam preferências alimentares e comportamento variado. Além disso, variáveis ambientais como, por exemplo, complexidade estrutural dos ecossistemas, temperatura e umidade, influenciam fortemente os resultados, considerando, por exemplo, que de acordo com Siqueira e Salomão (2016), a temperatura ideal para o desenvolvimento de esporos de Penicillium está em torno de $24^{\circ} \mathrm{C}$, sendo seu crescimento considerado lento em temperaturas acima de $35^{\circ} \mathrm{C}$ e abaixo de $10^{\circ} \mathrm{C}$.

\section{CONCLUSÕES}

O método de controle biológico que utiliza a calda diluída de macerados de laranjas em estágio avançado de decomposição cobertos por fungos Penicillium spp. demonstrou, no presente estudo, potencial de controle na atividade de formigas cortadeiras. Além disso, a cooperação dos agricultores na observação e obtenção de dados mostrou-se adequada aos resultados obtidos, visto que isto aumenta a confiança dos agricultores no método, e a transferência de conhecimentos entre agricultores tem maior capilaridade quando comparada ao conhecimento produzido apenas pelo meio acadêmico. Isto demonstra a importância de aprofundar este conhecimento de modo aliado à experimentação técnico-científica.

\section{REFERÊNCIAS}

BACCARO, B. F.; FEITOSA, R. M.; FERNANDEZ, F.; FERNDANDES, I. O.; IZZO, T. J.; DE SOUZA, J. L. P. Guia para os gêneros de formigas do Brasil. Manaus: Editora INPA, p. 388, 2015.

BALLESTER, A. R.; LAFUENTE, M. T. LED Blue Light-induced changes in phenolics and ethylene in citrus fruit: Implication in elicited resistance against Penicillium digitatum infection. Food chemistry, v. 218, p. 2017. https://doi.org/10.1016/j.foodchem.2016.09.089. Acesso em: 19 out 2019.

BAYLÃO JUNIOR, H. F., CARVALHO, D. C., CONDE, M. M. S., LORENZON, M. C., MAIMON, Z. L., \& GOMES, A. M. Plantas visitadas por Apoidea (Hymenoptera) na região de Cacaria, Município de Piraí-RJ. Revista Brasileira de Biociências, v.5 n.2, p. 1110-1112. 2008. Disponível em: $\quad \underline{\text { https://scholar.google.com.br/scholar?hl=pt- }}$ BR\&as_sdt=0\%2C5\&q=Plantas+visitadas + por + Apoidea $+\% 28$ Hymenoptera $\% 29+$ na + regi $\% \mathrm{C}$ 
Acesso em: 01 out 2020.

BERALDO, K. A.; RODRIGUES, M. G.; RODRIGUES, W. Núcleos de Estudos em Agroecologia: uma política pública para o fortalecimento da extensão universitária. Revista do Programa de Pós-Graduação em Extensão Rural (UFV), v. 7, n. 1, p. 398-416, 2018. Disponível em: https://www.semanticscholar.org/paper/N\%C3\%BAcleos-de-Estudos-emAgroecologia\%3A-uma-pol\%C3\%ADtica-o-Beraldo-

Mendon\%C3\%A7a/70669441882b92bf931fd45707df66b9df36e14d?p2df. Acesso em: 01 out 2020.

BUENO, I. Dinâmica do crescimento do fungo simbionte e estruturação de castas em formigas-cortadeiras (Hymenoptera: Formicidae). 2020. Dissertação (Mestrado em Entomologia) - Escola Superior de Agricultura Luiz de Queiroz, University of São Paulo, Piracicaba, 2020. Disponível em: https://www.teses.usp.br/teses/disponiveis/11/11146/tde07052020-094738/en.php. Acesso em: 19 ago 2019.

BURATTO, D. A., SOUZA, N. J., SOUZA, M. D., ROLIN, F. A. Resistance of bait granulated distributed in bulk and bait holder to action of humidity in areas of to action of moisture in Pinus taeda planting in southern highlands Catarinense. Ciência Florestal, v. 27, n. 3, p. 1083-1093, 2017. Disponível em: http://dx.doi.org/10.5902/1980509828683. Acesso em: 01 out 2020 .

CANALI, M. C. Prospecção de fungos entomopatogênicos para o controle biológico de formigas cortadeiras. 2017. 117f. Dissertação (Mestrado em Ciências Biológicas) Universidade Estadual Paulista, Instituto de Biociências de Rio Claro, Rio claro, 2017. Disponível

em: https://repositorio.unesp.br/bitstream/handle/11449/150198/canali_mc_me_rcla_int.pdf?seque nce $=6 \&$ isAllowed $=\mathrm{y}$. Acesso em: 01 out 2020.

CHAMY, M. N. C. L. Identificação de fungos produtores de enzimas extracelulares de interesse biotecnológico associados às formigas cortadeiras Atta sexdens (Linnaeus, 1758). 2017. 72 f. Dissertação (Mestrado em Biotecnologia) - Universidade Federal do Amazonas, $\quad 2017 . \quad$ Danaus, em: https://tede.ufam.edu.br/bitstream/tede/6257/5/Disserta\%c3\%a7\%c3\%a3o_Michel\%20N.\%20 \%20L.\%20Chamy.pdf . Acesso em: 01 out 2020. 
CAPORAL, R. R. Extensão Rural e Agroecologia: Temas Sobre um Novo Desenvolvimento Rural, Necessário e Possível. Brasília, 2009.

CASA, J., CARISSIMI BOFF, M. I., DRESH RECH, T., BOFF, P. Resistência do vimeiro, Salix spp.(Salicaceae), a pragas e doenças. Ciência Florestal, v. 17, n. 1, 2007. http://dx.doi.org/10.5902/198050981929. Disponível em: https://periodicos.ufsm.br/cienciaflorestal/article/view/1929/1171. Acesso em: 01 out 2020.

COELHO, F. M. G. A Arte das Orientações Técnicas no Campo. Editora UFV, p. 67 $100,2005$.

COME, S. F. Colocando os últimos em primeiro lugar: Análise da participação dos agricultores na pesquisa agrária e na extensão rural no distrito de Sussundega, Moçambique. 2019. 149 f. Tese (Doutorado em Extensão Rural). Universidade Federal de Viçosa, Viçosa, MG, Brasil. 2019. Disponível em: https://www.locus.ufv.br/bitstream/handle/123456789/27570/texto\%20completo.pdf?sequenc e=1\&isAllowed=y . Acesso em: 02 out 2020.

DE BRITTO, J.S., FORTI, L. C., DE OLIVEIRA, M. A., ZANETTI, R., WILCKEN, C. F., ZANUNCIO, J. C., DA SILVA CAMARGO, R. Use of alternatives to PFOS, its salts and PFOSF for the control of leafcutting ants Atta and Acromyrmex. International Journal of Research in Environmental Studies, v.3, p.11-92, 2016. Disponível em: https://www.researchgate.net/profile/Jose_Zanuncio/publication/303364980_Use_of_alternati ves_to_PFOS_its_salts_and_PFOSF_for_the_control_of_leafcutting_ants_Atta_and_Acromyrmex/links/59e111f50f7e9b97fbe2b34c/Use-of-alternativesto-PFOS-its-salts-and-PFOSF-for-the-control-of-leaf-cutting-ants-Atta-andAcromyrmex.pdf.Acesso em: 02 out 2020.

DA SILVA ARAÚJO, M., RODRIGUES, C. A., DE OLIVEIRA, M. A., DE JESUS, F. G. Controle biológico de formigas-cortadeiras: o caso da predação de fêmeas de Atta spp. por Canthon virens. Revista de Agricultura Neotropical, v. 2, n. 3, p. 8-12, 2015. Disponível em: https://periodicosonline.uems.br/index.php/agrineo/article/view/273/398 Acesso em: 02 out 2020.

DA SILVA, F. N. L.; PEREIRA, A. S.; DE ARAUJO OLIVEIRA, L. A.; DE OLIVEIRA, L. C.; Macedo, A. R. G.; DE QUAdroS, M. L. A.; DE SOUZA CASTRO, N. M. Metodologias de extensão rural aplicadas à aquicultura: um paralelo entre teoria e prática. 
Research, Society and Development, v. 9, n. 8, p. e245984168-e245984168, 2020. Disponível em: https://rsdjournal.org/index.php/rsd/article/view/4168/4758. Acesso em: 02 out 2020.

DO NASCIMENTO, M. O.; DE ALMEIDA SARMENTO, R.; DOS SANTOS, G. R.; DE OLIVEIRA, C. A.; DE SOUZA, D. J. Antagonism of Trichoderma isolates against Leucoagaricus gongylophorus (Singer) Möller. Journal of basic microbiology, v. 57, n. 8, p. 699-704, 2017.

Disponível

em: https://onlinelibrary.wiley.com/doi/abs/10.1002/jobm.201600755 . Acesso em: 02 out 2020.

DORNELAS, A. S. P., ALMEIDA SARMENTO, R., DOS SANTOS, G.R., NASCIMENTO, M. O., SOUZA, D. J. Fungos Filamentosos Associados às Espécies Atta sexdens (Linnaeus) e Atta laevigata (F. Smith) (Hymenoptera: Formicidae). EntomoBrasilis, v. $9, \quad$ n. $1, \quad$ p. 26-30, 2016. Disponível em: https://www.entomobrasilis.org/index.php/ebras/article/view/528 . Acesso em: 02 out 2020.

DORNELAS, A. S. P.; SARMENTO, R. A.; PEDRO-NETO, M.; SILVA, D. G.; SANTOS, G. R.; NASCIMENTO, M. O.; OLIVEIRA, C. A.; SOUZA, D. J. Susceptibility of Atta sexdens worker ants treated with the immunosuppressant Sandimmun Neoral to Metarhizium anisopliae. Pesquisa Agropecuaria Brasileira, v. 52, p. 133-136, 2017. Disponível em: $\quad$ https://www.scielo.br/scielo.php?pid=S0100204X2017000200133\&script=sci_arttext. Acesso em: 02 out 2020.

FONSECA, L. Z. Formigas em cavernas ferruginosas da Amazônia: indicadoras de diversidade e fatores que as influenciam. 2019. 42 p. Dissertação (Mestrado em Ecologia Aplicada)-Universidade Federal de Lavras, Lavras, 2019. Disponível em: http://177.105.2.222/bitstream/1/33235/1/DISSERTA\%C3\%87\%C3\%830_Formigas\%20em \%20cavernas\%20ferruginosas\%20da\%20Amaz\%C3\%B4nia\%20indicadoras\%20de\%20diver sidade \%20e\%20fatores\%20que\%20as\%20influenciam.pdf. Acesso em: 04 out 2020.

FREITAS, A., SOUZA, A. Q. L., MAKI, C. S., PEREIRA, J. O., SILVA, N. M. Atividade antagonista de bactérias endofíticas de plantas da Amazônia contra o fungo simbionte $L$. gongylophorus, e dos fungos associados presentes nos ninhos de Atta sexdens. Scientia Amazonia, v. 5, n. 1, p. 1-14, 2016. Disponível em: http://scientia-amazonia.org/wpcontent/uploads/2016/06/v5-n1-1-14-2016.pdf. Acesso em: 04 out 2020.

GIESEL, A., BOFF, P., BOFF, M. I. C., \& FERNANDES, P. Occurrence of the leafcutters ants in the high-altitude grasslands in the south Brazil. Research, Society and 
Development, $\quad$ n. $9, \quad$ v. $\quad 8, \quad 2020 . \quad$ Disponível em: https://www.rsdjournal.org/index.php/rsd/article/view/6365/5928 . Acesso em: 04 out 2020.

GIESTAS, P. H. C.; LACERDA, F. G.; CARMASSI, G. Forrageamento de operárias de Atta laevigata (hymenoptera: formicidae) sob efeito de Canavalia ensiformis (1.) dc (fabaceae). Biodiversidade, $\quad$ v. $19, \quad$ n. $1, \quad 2020 . \quad$ Disponível em: https://periodicoscientificos.ufmt.br/ojs/index.php/biodiversidade/article/view/9998. Acesso em: 05 out 2020.

HU, W.; ZHANG, N.; CHEN, H.; ZHONG, B.; YANG, A.; KUANG, F.; CHUN, J. Fumigant Activity of Sweet Orange Essential Oil Fractions Against Red Imported Fire Ants (Hymenoptera: Formicidae). Journal of Economic Entomology, p. 120, 2017. Disponível em: https://academic.oup.com/jee/article-abstract/110/4/1556/3746973. Acesso em: 05 out 2020.

JUNIOR, J. C. A. Concepções Pedagógicas e Modelos Históricos de Extensão Rural: uma Análise da ATER Paranaense. Revista Espaço Acadêmico, n. 225, 2020.

LIMA, M. S. C. A rede ATER Nordeste: Participação e conflitos de interesses no processo de implementação da Política Nacional de Assistência Técnica e Extensão Rural (PNATER). 2018. 230 f. Tese (Doutorado em Gestão de Sistemas) - Universidade Estadual de Campinas, Campinas, 2018. Disponível em: http://repositorio.unicamp.br/bitstream/REPOSIP/333222/1/Lima_MarinaDeSaCosta_D.pdf Acesso em: 25 jan 2021.

LINS, M. L.; PIRES, S. Velhas Alianças e Novos Compromissos: Extensão Rural e Cooperativismo Agrícola no Brasil. Revista de Extensão e Estudos Rurais, v. 7, n. 1, p. 118 140, 2018. Disponível em: https://periodicos.ufv.br/rever/article/view/3360. Acesso em: 05 out 2020.

MEIRELlES, L. A.; SOLOMON, S. E.; BACCI, M.; WRIGHT, A. M.; MUELLER, U. G. ; RODRIGUES, A. Shared Escovopsis parasites between leaf-cutting and non-leaf-cutting ants in the higher attine fungus-growing ant symbiosis. Royal Society Open Science, v. 2, p. 150-257, 2015. Disponível em: https://royalsocietypublishing.org/doi/10.1098/rsos.150257. Acesso em: 05 out 2020.

MOREIRA, S. S., TALACZ, V. Controle biológico de formigas cortadeiras com Penicilium sp. obtido da casca de laranja em decomposição. Cadernos de Agroecologia, [S.1.], 
v. $8, \quad$ n. 2, dec. 2013. Disponível em: http://revistas.abaagroecologia.org.br/index.php/cad/article/view/14661. Acesso em: 05 out 2020.

NAGAmOto, N. S., BARBIERI, R. F., FORTI, L. C., CARDOSO, S. R. D. S., MOREIRA, S. M., LOPES, J. F. S. Attractiveness of copperleaf-based bait to leaf-cutting ants. Ciência Rural, v. 41, n. $\quad 6$, p. 931-934, 2011. Disponível em: https://www.scielo.br/scielo.php?pid=S0103-84782011005000070\&script=sci_arttext. Acesso em: 05 out 2020.

NUNES, F. A., ROPPA, C., MEJIA, R. S., SOUZA, M. S. Núcleo de Estudos e Atuação em Agroecologia (NEAA) e sua abordagem perante grupo de agricultores familiares organizados. In: VI CONGRESSO LATINO-AMERICANO DE AGROECOLOGIA; X CONGRESSO BRASILEIRO DE AGROECOLOGIA.; V SEMINÁRIO DE AGROECOLOGIA DO DF E ENTORNO 2017, IFRJ Campus Pinheiral, RJ. Anais... Brasília - DF, 2017.

OI, D., PORTER, S., VALLES, S. A Review of the Biological Control of Fire Ants. Myrmecological News, v. 21, p. 101-116, 2015.

OLIVEIRA, M. T. B. A ambigüidade da extensão rural universitária e as acusações de técnicos. Revista de Economia e Sociologia Rural, v. 31, n. 2, p. 103-124, 2020. Disponível em: https://www.revistasober.org/article/5e9366e50e88256418dafd06/pdf/resr-31-2-103.pdf. Acesso em: 05 out 2020.

PALMA, A. M. C. Iscas biológicas para controle de formigas cortadeiras (Hymenoptera: Formicidae). 2016. 42f. Trabalho de Conclusão de Curso (Graduação em Engenharia Florestal) - Universidade Federal do Recôncavo da Bahia, Cruz das Almas, 2016. Disponível em: http://www.repositoriodigital.ufrb.edu.br/bitstream/123456789/949/1/TCC\%20\%20Adla\%20Palma.pdf . Acesso em: 05 out 2020.

POLEZEL, D. R. Fungos isolados de ninhos iniciais da formiga Atta sexdens rubropilosa: análise do potencial para biocontrole de formigas cortadeiras. 2017. 63p. Dissertação (Mestrado) - Universidade Estadual Paulista, Instituto de Biociências de Rio Claro, Rio claro, 2017. Disponível em: https://repositorio.unesp.br/bitstream/handle/11449/150431/polezel_dr_me_rcla_int.pdf?sequ ence $=6 \&$ is Allowed $=\mathrm{y}$. Acesso em: 05 out 2020. 
SANTOS, G. H. M. Características bioecológicas de Acromyrmex rugosus (Smith, 1858) e parasitismo pelo forídeo Apocephalus sp. 2017. 58f. Dissertação (Mestrado em Manejo e Conservação de Ecossistemas Naturais e Agrários) - Universidade Federal de Viçosa, Florestal - MG, 2017. Disponível em: https://www.locus.ufv.br/handle/123456789/11700 . Acesso em: 05 out 2020.

SCHNEIDER, I. A. Transferência de conhecimento agrícola num modelo reverso de comunicação. Revista de Economia e Sociologia Rural, v. 21, n. 2, p. 219-239, 2020. Disponível em: < https://www.revistasober.org/article/5ea962c00e88255327bd4789/pdf/resr21-2-219.pdf >. Acesso em: 05 out 2020.

SIQUEIRA, D. L., SALOMÃO, L. C. C. Citros: do Plantio à colheita. $1^{\circ}$ ed. Viçosa: Editora UFV, 2016, p. 182-184.

SILVA, B. L. Controle da formiga cortadeira (Atta sexdens rubropilosa) em agricultura orgânica no bioma Cerrado. 2017. 20f. Trabalho de Conclusão de Curso (Graduação em Agronomia) - Universidade Federal de Santa Catarina, Florianópolis, 2017. Disponível em: https://repositorio.ufsc.br/bitstream/handle/123456789/174309/TCC-

BRUNO\%20LUIZ\%20DA\%20SILVA.pdf? sequence=1\&isAllowed=y . Acesso em: 05 out 2020.

SILVA, B. C.; COSTA, E. C., SALDANHA, M. A., PROCKNOW, D., DE SOUZA, P. D., CRODA, J. P., \& CAPITANI, L. C. Métodos de controle e prevenção de insetos-praga em povoamentos florestais. Brazilian Journal of Development, v. 6, n. 7, p. 48477-48496, 2020. Disponível em: https://www.brazilianjournals.com/index.php/BRJD/article/view/13501/11317 . Acesso em: 05 out 2020.

SERRÃO, A. C. P. Em Tempos de Exceção como Fazer Extensão? Reflexões sobre a Prática da Extensão Universitária no Combate à COVID-19. Revista Práticas em Extensão, v. 04, n. 01, 2020.

Recebido em: 05 de outubro de 2020. Aceito em: 09 de março de 2021. 